\title{
HIGHER ORDER SINGULARITIES OF MORPHISMS TO PROJECTIVE SPACE
}

\author{
JOHAN P. HANSEN
}

\begin{abstract}
This paper proves existence theorems for higher order singularities of a finite morphism to $\mathbf{P}^{m}$ and deduces a result on simple connectivity of varieties admitting a finite morphism of bounded singularity.

The singularities are obtained by successive degeneration of double points of $f$. Our main tool is R. Schwarzenberger's notion of generalized secant sheaves and the connectedness theorem obtained by W. Fulton and the author.
\end{abstract}

Conventions. Throughout, varieties will be complete-possibly reducible and singular, unless mentioned otherwise-and defined over an algebraically closed field $k$ of arbitrary characteristic.

When we say that a variety is connected, it includes the assertion that it is nonempty.

We thank W. Fulton for several helpful discussions.

1. Higher order singularities. For a finite morphism $f: X \rightarrow Y$ of irreducible varieties, we say that a closed point $x \in X$ is a $q$ th order singularity if

$$
\operatorname{dim}_{k}\left(\mathcal{O}_{X, x} / f^{*} m_{f(x)}+m_{x}^{q+1}\right) \geqslant q+1 \text {, }
$$

where $\mathcal{O}_{X, x}$ is the local ring of $X$ at $x$ and $m_{X}$ (resp. $m_{f(x)}$ ) is the maximal ideal at $x$ (resp. $m_{f(x)}$ ). In this notion every point of $X$ is a 0th order singularity, and a first order singularity is a ramification point of $f$. Let $S^{q}=S^{q}(f)$ denote the set of $q$ th order singularity points.

If $\mathscr{P}_{X / Y}^{q}$ denotes the coherent $\mathcal{O}_{X}$-algebra of $q$ th order relative principal parts, there is an isomorphism

$$
\mathscr{P}_{X / Y}^{q} \otimes_{\mathcal{O}_{X}} k(x) \simeq \mathcal{O}_{X, x} / f^{*} m_{f(x)}+m_{x}^{q+1}
$$

for any closed point $x$ of $X$ [EGA, IV.16.4.5 and 16.4.12]. Therefore

$$
S^{q}=\left\{x \in X \mid \operatorname{dim}_{k}\left(\mathscr{P}_{X / Y}^{q}(x)\right) \geqslant q+1\right\}
$$

constitutes the $q$ th order singularity locus. By semicontinuity of the dimension of the fibres of a coherent sheaf, $S^{q}$ is a closed subscheme of $X$.

In the following, let

$$
\sigma_{q}=\operatorname{dim}(X)-q(\operatorname{dim}(Y)-\operatorname{dim}(X)+1),
$$

the expected dimension of $S^{q}$.

Received by the editors March 5, 1985 and, in revised form, April 30, 1985.

1980 Mathematics Subject Classification. Primary 14E20, 14E22, 14E25, 14 M07. 
In fact, if $Y$ is nonsingular, then every irreducible component of $S^{q}$, containing a smooth point $x$ of $X$, at which the map on Zariski tangent spaces

$$
(d f)_{x}: T_{X, x} \rightarrow T_{Y, f(x)}
$$

has rank $\geqslant \operatorname{dim}(X)-1$, has dimension $\geqslant \sigma_{q}[\mathbf{R}, 3.8]$.

Smooth points of $X$ in $S^{q}$, where the map on Zariski tangent spaces $(d f)_{x}$ : $T_{X, x} \rightarrow T_{Y, f(x)}$ has rank $\geqslant \operatorname{dim}(X)-1$, relate to the Thom-Boardman singularities $S_{1, \ldots, 1}$ ( $q$ indices) of a map of differentiable manifolds [T]. Namely assume $S^{q-1}$ is smooth at $x$ of dimension $\sigma_{q-1}$. Then the map on Zariski tangent spaces

$$
d\left(\left.f\right|_{S^{4-1}}\right)_{x}: T_{S^{4-1}, x} \rightarrow T_{Y, f(x)}
$$

has rank $=\operatorname{dim}\left(S^{q-1}\right)-1$ if and only if $x \in S^{q}(\operatorname{char} k=0)$ [R, 11.2].

2. Statements. Our existence theorem derives from the existence of degeneration of double points of $f$ at a point in $S^{q-1}$.

2.1. TheOREM. Let $X$ be an irreducible variety and $f: X \rightarrow \mathbf{P}^{m}$ a finite morphism. Fix $q$ with $\sigma_{q} \geqslant 0$. Assume $S^{q-1}$ has an irreducible component $V$ of dimension $\geqslant \sigma_{q-1}$, and the restriction of $f: f^{-1}(f(V)) \rightarrow F(V)$ is not one-to-one. Then $S^{q}$ is nonempty and has an irreducible component of dimension $\geqslant \sigma_{q}$.

2.2. Corollary. Let $X$ be a normal and irreducible variety. If $X$ admits a finite morphism $f: X \rightarrow \mathbf{P}^{m}$ with $S^{q}=\varnothing$ for some $q$ with $\sigma_{q} \geqslant 0$, then the fundamental group of $X$ is trivial.

The above assertions are proved in $\S 4$; an easy induction argument gives the following corollary.

2.3. Corollary. Let $X$ be an irreducible variety and $f: X \rightarrow \mathbf{P}^{m}$ a finite morphism with $\# f^{-1}(f(x)) \geqslant 2$ for all $x \in X$. Then $S^{q}$ is nonempty and has an irreducible component of dimension $\geqslant \sigma_{q}$ for all $q$ with $\sigma_{q} \geqslant 0$.

T. Gaffney [G] has obtained similar results with similar methods, using another definition of multiplicity.

For coverings of $\mathbf{P}^{m}$, Gaffney and R. Lazarsfeld obtained remarkable results [G-L]. Their main assertions are that there exist points with local degree $\geqslant$ $\min (d, m+1)$, where $d$ is the degree of the covering; and that a normal variety $X$, representable as a cover of $\mathbf{P}^{m}$ with degree $\leqslant m$, is algebraically simply connected.

P. Deligne [D] and W. Fulton (cf. [F-L]) show, using the topological version of the connectedness theorem for projective space, that $X$ is in fact topologically simply connected.

For a flat and generically étale covering, the local degree of [G-L] equals $\operatorname{dim}_{k}\left(\mathcal{O}_{X, x} / f^{*} m_{f(x)}\right)$. Clearly

$$
\operatorname{dim}_{k}\left(\mathcal{O}_{X, x} / f^{*} m_{f(x)}\right) \geqslant \operatorname{dim}_{k}\left(\mathcal{O}_{X, x} / f^{*} m_{f(x)}+m_{x}^{q+1}\right),
$$

and their existence assertion and simple-connectivity result are in accordance with 2.1 and 2.2 for coverings.

It should be observed that some assumption, such as normality, on $X$ is necessary in the $\pi_{1}$-result for coverings, as shown by an example of A. Landman. 
J. Roberts [R] studied higher order singularities of generic linear projections $X \rightarrow \mathbf{P}^{m}$.

3. Degeneration and generalized secant sheaves. In this section, we establish that degeneration of double points of $f$ at a point in $S^{q-1}$ gives rise to a point in $S^{q}$. Our main tool in proving this is Schwarzenberger's notion of generalized secant sheaves [Sc] and a result of $A$. Kato $[\mathbf{K}]$ relating these to the sheaves of relative principal parts.

3.1. Proposition. Let $f: X \rightarrow Y$ be a finite, projective morphism between irreducible varieties, and let $V$ be an irreducible component of $S^{q-1}(f)$. If $Z \neq \Delta_{V}$ is an irreducible component of $V \times{ }_{Y} X$, then

$$
\delta^{-1}\left(Z \cap \Delta_{V}\right) \subset S^{q}(f),
$$

where $\Delta_{V}$ is the image of the diagonal morphism $\delta: V \rightarrow V \times_{Y} X$.

Let $f: X \rightarrow Y$ be a finite, projective morphism, and let $X_{n}=X \times_{Y} \cdots \times_{Y} X$ denote the $n$-fold relative product of $X$ over $Y$.

Consider morphisms $h_{t}: X_{n} \rightarrow X_{n} \times_{Y} X$, where

$$
h_{t}:\left(x_{1}, \ldots, x_{n}\right) \rightarrow\left(x_{1}, \ldots, x_{n}\right) \times x_{t} .
$$

If $W_{n}$ (resp. $\tilde{W}_{n}$ ) denotes the union of the images of $h_{t}, t=1, \ldots, n$ (resp. $t=1, \ldots, n-1)$, then the two diagrams below constitute product schemes in the sense of $[\mathbf{S c}]$ :
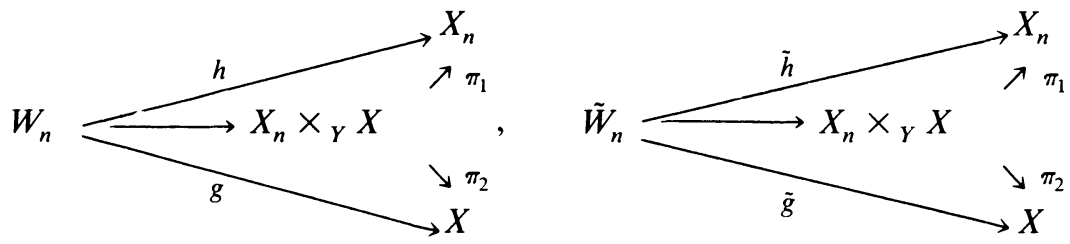

Let $\Sigma^{n}=h_{*} g^{*}\left(\mathcal{O}_{X}\right), \quad \tilde{\Sigma}^{n}=\tilde{h}_{*} \tilde{g}^{*}\left(\mathcal{O}_{X}\right)$ denote the associated generalized secant sheaves which are coherent [EGA, III.2.2.1].

3.2. LEMMA. With notation as above, the following holds:

(i) $\pi_{1}^{*}\left(\Sigma^{n}\right) \simeq \tilde{\Sigma}^{n+1}$, where $\pi_{1}$ is the projection $\pi_{1}: X_{n+1}=X_{n} \times_{Y} X \rightarrow X_{n}$,

(ii) If $\left(x_{1}, \ldots, x_{n}, x_{n+1}\right) \in W_{n} \backslash \tilde{W}_{n}$, then $\operatorname{dim}_{k(\mathbf{x})}\left(\Sigma^{n}(\mathbf{x})\right)>\operatorname{dim}_{k(\mathbf{x})}(\tilde{\Sigma}(\mathbf{x}))$, where $\mathbf{x}=\left(x_{1}, \ldots, x_{n}\right) \in X_{n}$. by

Proof. A. Kato [K, p.103] establishes (i). To obtain (ii), let $\mathscr{J}$ be the ideal defined

$$
0 \rightarrow \mathscr{J} \rightarrow \mathcal{O}_{W_{n}} \rightarrow \mathcal{O}_{\tilde{W}_{n}} \rightarrow 0 .
$$

Since $h$ is finite, we need only show that $\mathscr{J}_{\left(x_{1}, \ldots, x_{n+1}\right)} \neq 0$, which follows from our assumption.

3.3. Lemma (A. Kato [K, 3.3]). If $\Delta: X \rightarrow X_{n+1}$ is the diagonal morphism, then $\Delta^{*}\left(\Sigma^{n+1}\right) \simeq \mathscr{P}_{X / Y}^{n}$. 
Proof of 3.1. Adopting notation as in the proposition, let $z \in \delta^{-1}\left(Z \cap \Delta_{V}\right)$. We can find a curve $T$ and a morphism $c: T \rightarrow Z$, with $c\left(t_{0}\right)=(z, z)$ for some $t_{0} \in T$ and $c(t) \notin \Delta_{V}$ for $t \in T \backslash\left\{t_{0}\right\}$. Projecting onto the factors of $V \times{ }_{Y} X$ yields morphisms $c_{1}, c_{2}: T \rightarrow X$.

Consider

$$
\tilde{c}(t)=\left(c_{1}(t), \ldots, c_{1}(t), c_{2}(t)\right) \in X_{q+1},
$$

with $\tilde{c}\left(t_{0}\right)=(z, \ldots, z)$. For $t \neq t_{0}$,

$$
\tilde{c}(t) \times c_{1}(t) \in W_{q+1} \backslash \tilde{W}_{q+1} .
$$

Hence by 3.2(ii)

$$
\operatorname{dim}_{k(\tilde{c}(t))}\left(\Sigma^{q+1}(\tilde{c}(t))\right)>\operatorname{dim}_{k(\tilde{c}(t))}\left(\tilde{\Sigma}^{q+1}(\tilde{c}(t))\right) .
$$

By 3.2(i), 3.3 and assumption on $V$, we get that

$$
\operatorname{dim}_{k(\tilde{c}(t))}\left(\Sigma^{q+1}(\tilde{c}(t))\right) \geqslant q+1
$$

for $t \neq t_{0}$. Finally we conclude by semicontinuity of the dimension of the fibres of a coherent sheaf, and by 3.3 , that

$$
\operatorname{dim}_{k(z)}\left(\mathscr{P}_{X / Y}^{q}(z)\right) \geqslant q+1 .
$$

\section{Proofs of main results.}

Proof of 2.1. Let $f: X \rightarrow \mathbf{P}^{m}$ be a finite morphism and $V$ an irreducible component of $S^{q-1}$ with $\operatorname{dim}(V) \geqslant \sigma_{q-1}$. Consider the finite morphism

$$
F=f_{\mid V} \times f: V \times X \rightarrow \mathbf{P}^{m} \times \mathbf{P}^{m},
$$

where $V \times X$ is irreducible and $\operatorname{dim}(V \times X) \geqslant \sigma_{q-1}+\operatorname{dim}(X)$. By 5.5(i), $F^{-1}\left(\Delta_{\mathbf{p}^{m}}\right)$ is $\left(\sigma_{q-1}+\operatorname{dim}(X)-m-1\right)$-connected. By assumption $\Delta_{V}$ is not the only irreducible component of $F^{-1}\left(\Delta_{\mathbf{p}^{m}}\right)$, so there must be an irreducible component $Z \neq \Delta_{V}$ of $F^{-1}\left(\Delta_{\mathbf{P}^{m}}\right)$ with

$$
\operatorname{dim}\left(Z \cap \Delta_{V}\right) \geqslant \sigma_{q-1}+\operatorname{dim}(X)-m-1,
$$

and we are finished by 3.1 , because the right side above is equal to $\sigma_{q}$.

Proof of 2.2. For $f: X \rightarrow \mathbf{P}^{m}$ as in the corollary, let $q$ be maximal so that $S^{q}$ has an irreducible component $V$ of dimension $\geqslant \sigma_{q}$. By assumption, $\sigma_{q+1} \geqslant 0$.

Let $\pi: V^{*} \rightarrow V$ be the normalization of $V$ and consider

$$
(f \circ \pi) \times f: V^{*} \times X \rightarrow \mathbf{P}^{m} \times \mathbf{P}^{m} .
$$

Our main theorem (2.1) tells us-by maximality of $q$-that the restriction of $f$ : $f^{-1}(f(V)) \rightarrow f(V)$ is one-to-one, and therefore

$$
((f \circ \pi) \times f)^{-1}\left(\Delta_{\mathbf{p}^{m}}\right) \simeq V^{*} .
$$

The topological version of the connectedness theorem for projective space [D] (cf. [F-L]) now implies that the homomorphism $\pi_{1}\left(V^{*}\right) \rightarrow \pi_{1}\left(V^{*} \times X\right)$ is a surjection, which forces $\pi_{1}(X)$ to be trivial. 


\section{An $i$-connectedness theorem for projective space.}

5.1. Definition. Fix $l \geqslant 0$. A variety $X$ is said to be $l$-connected if all its irreducible components have dimension greater or equal to $l+1$, and for all its closed subvarieties $V$, with $\operatorname{dim}(V)<l, X \backslash V$ is connected.

With this definition, 0 -connected means that all irreducible components of $X$ are at least curves and $X$ is connected. It is important to note that an irreducible variety $X$ of dimension $\geqslant 1$ is $(\operatorname{dim}(X)-1)$-connected but not $\operatorname{dim}(X)$-connected.

The following definition and lemma provide another useful and perhaps more intuitive way of viewing this notion.

5.2. Definition. A sequence $Z_{0}, \ldots, Z_{n}$ of closed, irreducible subvarieties of $X$, all of dimension greater than or equal to $l+1$, with $\operatorname{dim}\left(Z_{i-1} \cap Z_{i}\right) \geqslant l$ for all $i=1, \ldots, n$, will be called an $l$-join within $X$ and $Z_{0}, Z_{n}$ are referred to as the extreme components.

5.3. LEMMA. A variety $X$ is l-connected if and only if any two irreducible components of $X$ are the extreme components of an l-join within $X$. Equivalently, $X=Z_{0}$ $\cup \cdots \cup Z_{n}$ for some l-join $Z_{0}, \ldots, Z_{n}$.

Proof. Assume $X=Z_{0} \cup \cdots \cup Z_{n}$, where $Z_{0}, \ldots, Z_{n}$ is an $l$-join within $X$, and let $V$ be a closed subvariety of $X$ with $\operatorname{dim}(V)<l$. Then $X \backslash V=\left(Z_{0} \backslash V\right)$ $\cup \cdots \cup\left(Z_{n} \backslash V\right)$, and $Z_{i} \backslash V$ are irreducible and nonempty for all $i=0, \ldots, n$ by reason of dimension. Likewise, $\left(Z_{i-1} \backslash V\right) \cap\left(Z_{i} \backslash V\right)=\left(Z_{i-1} \cap Z_{i}\right) \backslash V$ are nonempty for all $i=1, \ldots, n$. We conclude that $X \backslash V$ is connected.

Next assume that $X$ is $l$-connected. Let $X^{\prime}$ be the union of all irreducible components of $X$, which can be $l$-joined to a given irreducible component $Z^{\prime}$, and let $X^{\prime \prime}$ be the union of the other irreducible components. By definition of $X^{\prime}$ and $X^{\prime \prime}, \operatorname{dim}\left(X^{\prime} \cap X^{\prime \prime}\right)<l$; hence $X \backslash\left(X^{\prime} \cap X^{\prime \prime}\right)$ is connected; i.e., $X^{\prime}=X$.

5.4. LemMA. Let $X_{0}, \ldots, X_{s}$ be l-connected varieties, and assume $\operatorname{dim}\left(X_{i-1} \cap X_{i}\right)$ $\geqslant l$ for all $i=1, \ldots, s$. Then the union $X_{0} \cup \cdots \cup X_{s}$ is l-connected.

Proof. By assumption we can find irreducible components $Z_{i-1}$ and $Z_{i}^{\prime}$ of $X_{i-1}$ and $X_{i}$ such that $\operatorname{dim}\left(Z_{i-1} \cap Z_{i}^{\prime}\right) \geqslant l$ for all $i=1, \ldots, s$. Since $X_{i}$ is $l$-connected, 5.3 allows us to display $X_{i}$ as an $l$-join, which we, without restriction, can assume has $Z_{i}^{\prime}$ and $Z_{i}$ among its extreme components. The union of all the $l$-joins then constitutes an $l$-join exhausting $X$, which therefore by 1.3 must be $l$-connected.

5.5. TheOREM. Let $P=\mathbf{P}^{m} \times \cdots \times \mathbf{P}^{m}$ be a product of $r$ copies of projective $m$-space, and let $\Delta$ be the image of the diagonal embedding of $\mathbf{P}^{m}$ in $P$. Let $d=\operatorname{codim}(\Delta, P)=(r-1) m$. Assume $X$ is complete and l-connected, $l \geqslant d$, and consider a morphism $f: X \rightarrow P$.

(i) If $f$ is finite, then $f^{-1}(\Delta)$ is $(l-d)$-connected.

(ii) If for all $p \in P \backslash \Delta, \operatorname{dim}\left(f^{-1}(p)\right) \leqslant l-d$, then $f^{-1}(\Delta)$ is 0 -connected.

Before proceeding to the proof, recall the following properties enjoyed by projective space, of which (ii) is the connectedness theorem of $[\mathbf{F}-\mathbf{H}]$ and (i) is another way of saying that subvarieties of complementary dimension in projective space must meet. 
5.6. Proposition. Let $P=\mathbf{P}^{m} \times \cdots \times \mathbf{P}^{m}$ be the product of $r$ copies of projective $m$-space, and let $\Delta$ be the image of the diagonal embedding of $\mathbf{P}^{m}$ in $P$. For any morphism $f: X \rightarrow P$ from an irreducible and complete variety $X$ :

(i) $f^{-1}(\Delta) \neq \varnothing$ if $\operatorname{codim}(f(X), P) \leqslant m$.

(ii) $f^{-1}(\Delta)$ is connected if $\operatorname{codim}(f(X), P)<m$.

Proof OF 5.5. (i) To begin with, assume that $X$ is irreducible of dimension $\geqslant l+1$, $l \geqslant d$, and let $V \subset f^{-1}(\Delta)$ be a closed subvariety, such that $f^{-1}(\Delta) \backslash V$ is disconnected. We must show that $\operatorname{dim}(V) \geqslant l-d$. Assuming this is not the case, we can choose a linear subspace $L \subset \Delta$ of codimension $l-d$, such that $V \cap f^{-1}(L)=\varnothing$. The image of every irreducible component of $f^{-1}(\Delta)$ has dimension $\geqslant l-d+1$; hence $f^{-1}(L)$ meets every irreducible component of $f^{-1}(\Delta)$. Therefore $f^{-1}(L)$ is disconnected. On the other hand, consider

$$
f \times i: X \times L \rightarrow \tilde{P}=P \times \mathbf{P}^{m},
$$

where $i: L \rightarrow \Delta \simeq \mathbf{P}^{m}$ is the inclusion. Then

$$
\begin{aligned}
\operatorname{codim}((f \times i)(X \times L), \tilde{P}) & =\operatorname{codim}(f(X), P)+l-d \\
& =\operatorname{dim}(P)-(l+1)+l-d<m ;
\end{aligned}
$$

therefore by 5.6(ii), $f^{-1}(L) \simeq(f \times i)^{-1}(\tilde{\Delta})$ is connected, contradicting our assumption.

In the general case, let $Z_{0}, \ldots, Z_{n}$ be an $l$-join with $X=Z_{0} \cup \cdots \cup Z_{n}$. Then $f^{-1}(\Delta)=W_{0} \cup \cdots \cup W_{n}, W_{i}=\left(f_{\mid Z_{i}}\right)^{-1}(\Delta)$ is $(l-d)$-connected by what we have just seen. By assumption $\operatorname{dim}\left(Z_{i-1} \cap Z_{i}\right) \geqslant l$ for all $i=1, \ldots, n$; hence $W_{i-1} \cap W_{i}$ is nonempty and of dimension $\geqslant l-d$ for all $i=1, \ldots, n$ by 5.6(i). Therefore we conclude by 5.4 that $f^{-1}(\Delta)$ is $(l-d)$-connected.

(ii) Our assumptions on the fiber dimensions imply that an irreducible component $Z$ of $X$ is either mapped to $\Delta$, or $\operatorname{codim}(f(Z), P)<m$. In either case $\left(f_{\mid Z}\right)^{-1}(\Delta)$ is 0 -connected, the latter case following from 5.6(ii), and the former being trivial. Now if $X=Z_{0} \cup \cdots \cup Z_{n}$ is an $l$-join, then for every $i=1, \ldots, n$, either $f\left(Z_{i-1} \cap Z_{i}\right)$ $\subset \Delta$ or $\operatorname{codim}\left(f\left(Z_{i-1} \cap Z_{i}\right), P\right) \leqslant m$. In both cases we conclude that $f\left(Z_{i-1} \cap Z_{i}\right)$ $\cap \Delta \neq \varnothing$; the first case is trivial, and the second follows from 5.6(i). Our assertion now follows from 5.4 , because $f^{-1}(\Delta)=W_{0} \cup \cdots \cup W_{n}$, where $W_{i}=\left(f_{\mid Z_{i}}\right)^{-1}(\Delta)$ is 0 -connected and $W_{i-1} \cap W_{i} \neq \varnothing$ for all $i=1, \ldots, n$.

\section{REFERENCES}

[D] P. Deligne, Le groupe fondamental du complement d'une courbe plane n'ayant des points doubles ordinaires est abélian, Séminaire Bourbaki, 1979/80, no. 543.

[EGA] A. Grothendieck and J. Dieudonné, Eléments de géométrie algébrique. II, III, IV, Inst. Hautes Etude Sci. Publ. Math. 11 (1961), 32 (1967).

[F-H] W. Fulton and J. Hansen, A connectedness theorem for projective varieties, with applications to intersections and singularities of mappings, Ann. of Math. (2) 110 (1979), 159-166.

[F-L] W. Fulton and R. Lazarsfeld, Connectivity and its applications in algebraic geometry, Lecture Notes in Math., vol. 862, Springer-Verlag, Berlin and New York, 1981, pp. 26-93.

[G] T. Gaffney, Multiple poiants and associate ramification loci, singularities, Proc. Sympos. Pure Math. vol. 40, parts 1, 2, Amer. Math. Soc., Providence, R.I., 1983, pp. 429-437. 
[G-L] T. Gaffney and R. Lazarsfeld, On the ramification of branched coverings of $\mathbf{P}^{m}$, Invent. Math. 59 (1980), 53-58.

[K] A. Kato, Singularities of projective embedding ( points of order $n$ on an elliptic curve), Nagoya Math. J. 45 (1971), 97-107.

[L] R. Lazarsfeld, Thesis, Brown University.

[R] J. Roberts, Singularity subschemes and generic projections, Trans. Amer. Math. Soc. 212 (1975), $229-268$.

[Sc] R. L. E. Schwarzenberger, The secant bundle of a projective variety, Proc. London Math. Soc. (3) 14 (1964), 369-384.

[T] R. Thom, Les singulafités des applications différentiables, Ann. Inst. Fourier (Grenoble) $6(1955 / 56)$, 43-87.

Matematisk Institut, Aarhus Universitet, Ny Munkegade, DK 8000 Aarhus C, Denmark 